\title{
The Application of Steel Structure in Civil Engineering
}

\author{
Xiaodan $\mathrm{Wei}^{1}$, Xiangrong $\mathrm{He}^{1}$ \\ ${ }^{1}$ School of Civil Engineering and Architecture, Southwest University of Science and Technology, \\ Mianyang, 621000, China
}

Keywords: Civil engineering. Steel structure. Building

\begin{abstract}
Concrete and steel are two indispensable important construction materials. As the rapidly economic development and urbanization proceeding, the construction industry is towards to high-end direction development. In high-rise buildings, the advantage of steel structure is obvious, and owns considerable economic and social benefits. This paper clarifies the main characteristic of the steel structure in civil engineering; analysis the basic principle that maintain the stability of steel structure; discusses the construction method of steel structure in civil engineering, and puts forward the development trend of steel structure in civil engineering.
\end{abstract}

\section{Introduction}

It has been more than 100 years since steel structure born in this world. As early as 19 century, the high-rise building in Chicago has applied steel structure. Since in 1960s, America began to design and construct the high-rise steel structure that more than 80 floors. By the turn of the century, the propositions of steel frame residences have reached more than $50 \%$ in the US housing market. For our country, as urbanization continuous development, residential has also become industrialization, therefore, it more easily applies steel structure. It can be seen that encouraging the development steel construction actively has become the only way to develop the construction industry in our country. Steel structure owns high strength and applies in civil engineering construction very common, thus, it has a very broad space for development.

\section{The main characteristic of steel structure in civil engineering}

\section{Strength characteristics}

In general, it's decided by the strength material about the structure bearing and holding effect ability. One can use the relevant national standards to promote the performance of structural steel components, there list materials that steel structure could use above standards, such as building shall meet CSA standard or ASTM standard. As the greatly change of construction in recent years, the relevant data of tensile strength and yield strength used in structure are all from the history record of CISC, and now Canada identified the 7 steel grade and 8 strength rank about engineering structural steel, and ordinary building structural steel, and the range of yield strength is from 260 to $700 \mathrm{Mpa}$. But not all steel own all strength rank, so, once it's a particular steel structure design, it would become extremely important for the choice of type and strength grade of steel. In general, using steel structure could reduce the consumption of material; reduce the weight of structure; shorten the support component and foundation size, finally, reduce the structure cost of building.

\section{Stiffness characteristics}

Many other applicability parameters, like deformation are decided by stiffness component, and decided by stiffness structure system. However, in brief, component stiffness are mainly decided by material characteristic geometric cross section and material elasticity modulus, elasticity modulus of steel structure is 200Gpa, while, the ordinary density compressive strength is in the range of 20-40Gpa. Elasticity modulus of concrete is in the range of 40-45Gpa. For this reason, the rigidity of steel structure is five times than concrete's, and it's significant.

\section{Ductility characteristic}

Ductility is mainly refers to a material owns a deformation capacity of non plastic fracture in the drawing process. In general, ductility is an important characteristic parameter in structure design, 
especially in earthquake design, like those buildings surviving in the earthquake depend on the hysteresis energy dissipation of the frame of main structure after experiencing inelastic deformation. It should be mentioned that steel structure has become the most widely used and best toughness engineering material. But, the internal ductility of material would not transform into internal ductility of steel structure. So, in order to fully recognize this characteristic, one should use reasonable design methods, reliable and stable hysteresis elimination system. In general, if a design wants to have ductility response, it should have enough material section, material ductility, structure ductility, and component ductility; however, ductility capability and demand should match for the ductility level, curvature ductility, and displacement ductility. But, even though steel structure has relatively high ductility, due to the unstable bending on the stress, therefore, ductility in component curvature tends to deficiency.

\section{Toughness characteristic}

Toughness is a main index to judge the capability of absorbing energy and plastic fracture before the material fracture. It could resist the unstable crack development of notch. In general, toughness refers that steel structure could bear relatively large industry deformation in manufacture, installation, and using process, this is also an important characteristic of steel structure. Because of the toughness, it reduces the possibility of crack in bending punching, forging and drilling. Steel structure should have adequately fracture toughness, especially to the buildings of alternating load and impact load. Steel structure fracture toughness is particular to about the temperature condition, and as the temperature decreases, it gradually reduced. Therefore, designing the steel structure building in cold land, one should consider the toughness first. Relatively speaking, low carbon niobium steel is more flexibility than high carbon steel component. From the overall point of view, no matter stiffness and strength, or ductility characteristic, steel structure should be used priority to steel concrete, and steel structure is easily to construct the exoticness. In general, steel structure could offer the most ideal design, flexibility and space utilization. According to the steel structure, another advantage is to have ideal construction system. Reasonable shipping uses hollow steel bracket and component to trepanning, so, they should offer the corresponding channel for pipe and some other lines, then, when in the process of construction, the structure construction slag that equipped in steel structure need to be discussed specially.

\section{The principles to maintain steel structure}

Stability is always an important problem in using steel structure. Among various types of steel structure, one often meets stability problems. Once the problem handled improperly, it would lead to loss. According to characteristic that stability in actual design, one should implement the following three principles, and ensure the component of steel structure stability design, and would not loss stability. First, layout of the whole structure must consider the stability requirements of the whole system and components. At present, the structure is based on plane system, like truss and frame. Ensure the plane structure wouldn't cause the instability problems; it needs to be solved from overall arrangement structure, that needs to design the necessary supporting artifacts, which means the plane stability calculation of plane structure component should consistent with the structure arrangement. Second, the brief calculation of steel structure should be consistent with practical brief calculation, this is very important for frame structure stability calculation. At present, in design single or multilayer frame structure, one used to take frame column stability calculation place of frame stability analyze. Applying this method, it should use the coefficient of column calculation length when calculate the stability of frame column, so that make column stability calculation has effect on frame stability calculation. But, the actual framework is diversification, in order to simplify the calculation in design, one need to set the corresponding condition. Third, design detail structure must cooperate with structure stability calculation, and keep consistency. Structure calculation and structure design should conform to each other, which raise attention in structure design. To whether passing bending moment connection, it should full amount of rigidity and flexibility each other, and reduce the eccentricity of bar for the truss nodes as soon as possible. 


\section{The construction method of steel structure in civil engineering}

\section{Attach importance to the method of selection and connection}

In general, we divided the steel material into sheet, profiles, metal products and pipe. Steel structure in civil engineering mainly uses common low alloy steel, carbon structural steel, and ordinary carbon steel material; this is decided by hardness of steel. Beam is the mainly material in welding, or rolling " $\mathrm{H}$ ” type, once customers have requirements, it could conform to the cross section. Before installation, one should implement weld test to main weld join, and determine the welding material and other kinds of parameters. For the connection between beam and beam, beam and column, it could use weld or high strength bolt to connect. One should fully pay attention to the accuracy of the high strength bolt connection. For drilling the hole, there are two mainly ways, one is the high precision $\mathrm{CNC}$ drilling, the other is the lower accuracy template drilling. If technical permits, one should use the multi-axis to drill. After the material shipped to the building site, one should inspect the bolt parameters. During the installation process, one shouldn't twist by using spanner, or score in by using hammer, the steps must be through the early twist, retwist, and finally twist.

\section{The storage method steel structure}

At present, in our country, the site area in universal implementation structure of implementation construction is based on 1.5 times of floor space to implementation, and pile up according to the installation process; turn the yard form and set to the steel component; use loading machinery to place it in the installation of radius of gyration.

\section{Paint smear method}

Implementing paint smear is one of the important steps in building steel structure construction; it can be mainly divided into the four steps to implement. First, carry out the basic processing. Before entering to the paint coated process, one should clean up the surface of metal completely, and remove the rust. Once the area is not large, one could clean up the rust by using artificial. However, if appearing corrosion phenomenon, one could use steel wire brush or shovel knife to clean when it's seriously, and if it has large area corrosion, one could clear up by using grinder. Second, use antirust paint to blot out. After processing the base, one should ensure the drying surface, and implement antirust paint to smear. One should be carefully, meticulous, and besmear homogeneous, especially should pay attention to the rivet hole shouldn't be painted. After antirust paint drying, one should use the putty that match for paint to shave the surface. In order to ascend hardness, one could add appropriate red lead power or paste paint. Third, use etches primer to smear. Etch primer is the main point of paint daub, after daubing etch primer several hours, one could implement primer daub, 24 hours after; clean up the residues phosphide in the surface. The complete standard of daub is fairly uniform phosphate film after the surface dried. Fourth, use the daub coating to paint. Finally, brush steel structure paint. When brush paint, the standard is for reason and brush, and ensure that the paint not flow and not fall, it's considerable technical difficulties work, and when daubed, one should ensure the color and light consistent with each other, During daubing, one should check in timely, or avoid loss. In general, one should besmear at least twice, while the thickness should be at least more than 70 microns.

\section{The development trend of steel structure in civil engineering}

In recent years, although the steel structure in our country already has a considerable development and utilization, as a whole, it's still in developed. According to the lowest level that western developed countries set to estimate, there are at least 36 million tons steels on the development. The point is that the steel structure research has entered into a new development phase, some relevant regulations and standards have been formulated, which offer great material and technical guarantee for the development of steel structure. Therefore, China would be more actively to develop steel structure building, strive make the use of construction steel reach more than $3 \%$ of the total steel production. In 2015, the amount of construction would reach at least $6 \%$ of the total production. So, the development of steel structure has a broad prospect. Later, on development of steel structure, one 
should pay attention to offshore oil, steel structure and steel structure bridge, among them, the ocean oil output will reach 100 million tons; marine engineering equipment enterprises need to provide the corresponding equipment for deep sea mining; steel structure housing must conform to the green, environmental protection, energy saving and reduction policy, steel structure standardization housing production would has more broad market for space development.

\section{Conclusions}

In a word, steel structure has important position in civil engineering. During the process of construction, one should use the advantage of steel structure actively; implement scientization, standardization construction, then promote the use of steel structure value better. This is because that steel structure housing is fit for the goal of housing industrialization in our country, suit for sustainable development strategy; it has considerable effect on protecting cultivated land resource and enhancing the level of urbanization. The author believes that steel structure industry has nice prospect for development under the support of national policy and technology.

\section{References}

[1] Xiao Honglian. Introduce the application of steel structure in civil engineering [J]. Science Technology Information, 2012 (3).

[2] Xu Chunbo. Analysis the characteristic of steel structure implementation in civil engineering [J].Technology Innovation and Application, 2012 (31)

[3] Zhou Changyu. Application of steel structure in civil engineering [J]. China New Technologies and Products, 2012 (14). 